\title{
Características de frutos de pimentão cultivado em ambiente protegido sob doses de nitrogênio via fertirrigação
}

\author{
Jucilene S. Araújo ${ }^{1}$, Alberício P. de Andrade ${ }^{2}$, Cícera I. Ramalho ${ }^{2}$ \& Carlos A. V. de Azevedo ${ }^{3}$
}

\begin{abstract}
RESUMO
Este trabalho foi conduzido no Centro de Ciências Agrárias-UFPB, de fevereiro a junho de 2004, com o objetivo de avaliar o efeito de doses de nitrogênio aplicadas via fertirrigação, sobre as características de frutos de pimentão cultivado em ambiente protegido. O delineamento experimental adotado foi o de blocos casualizados, com cinco tratamentos $\left(0,100,200,300\right.$ e $400 \mathrm{~kg} \mathrm{ha}^{-1}$ de N) e quatro repetições. Avaliaram-se comprimento, diâmetro e peso médio de frutos e número de frutos por planta. Constataram-se efeitos significativos das doses de nitrogênio sobre o diâmetro, peso médio e número de frutos total, comercial e não-comercial do pimentão. O comprimento de frutos do pimentão não foi influenciado pelas doses de nitrogênio; no entanto, o valor médio obtido $(82 \mathrm{~mm})$ está dentro do padrão para a variedade estudada. O número máximo de frutos comerciais por planta foi de 12,8, observado na dose de $400 \mathrm{~kg}_{\text {ha-1 }}{ }^{1}$ de nitrogênio, enquanto na testemunha ( $0 \mathrm{~kg} \mathrm{ha}^{-1}$ de N) este valor foi de 5,5, havendo um incremento de 7,3 (aumento de 133\%) frutos considerados de qualidade superior (comercial) em relação à testemunha. Os frutos reconhecidos como não-comerciais, apresentaram-se com excelente aspecto, em termos de aparência e qualidade sanitária, tendo apenas comprimento e diâmetro fora dos padrões preestabelecidos.
\end{abstract}

Palavras-chave: Capsicum annuum, adubação nitrogenada, estufa

\section{Characteristics of bell pepper fruits cultivated in greenhouse under doses of nitrogen via fertirrigation}

\begin{abstract}
This study was conducted at the Center of Agricultural Sciences-UFPB, from February to June 2004, aiming to evaluate the effect of applied doses of nitrogen via fertigation, on the characteristics of bell pepper fruits cultivated in greenhouse. The adopted experimental design was in randomized blocks, with five treatments $\left(0,100,200,300\right.$ and $\left.400 \mathrm{~kg} \mathrm{ha}^{-1}\right)$ and 4 repetitions. Length, diameter and mean weight of fruits and number of fruits per plant were evaluated. Significant effects of the doses of nitrogen were verified on the diameter, mean weight and total, commercial and noncommercial number of fruits of bell pepper. The length of fruits of the bell pepper was not influenced by the doses of nitrogen; however, the obtained values are within the standard average for the studied variety. The maximum dose of nitrogen $\left(400 \mathrm{~kg} \mathrm{ha}^{-1}\right)$ promoted an increment of 7.3 (increase of 133\%) fruits considered of commercial quality in relation to the control $\left(0 \mathrm{~kg} \mathrm{ha}^{-1}\right)$. The fruits recognized as noncommercial showed an excellent aspect, in their appearance and hygiene quality, with just their length and diameter below the estabilished standards.
\end{abstract}

Key words: Capsicum annuum, nitrogen manuring, greenhouse

INSA, Av. Floriano Peixoto 715, Centro, CEP 58100-001, Campina Grande, PB. Fone: (83) 2101-6412. E-mail: jucilenearaujo@insa.gov.br

${ }^{2}$ CCA/UFPB, Campus II, CP 22, CEP 58397-000, Areia, PB. Fone: (83) 3362-2300, Fone-Fax: (83) 3362-2259. E-mail: albericio@uol.com.br; isaramalho3@hotmail.com

3 UAEAg/UFCG, Av. Aprígio Veloso 882, Bodocongó, CEP 58429-140, Campina Grande, PB. Fone: (83) 3310-1056. E-mail: cazevedo@deag.ufcg.edu.br 


\section{INTRODUÇÃO}

A produção de olerícolas é uma atividade agrícola muito vantajosa quando praticada em épocas adequadas, locais de boas condições climáticas e de mercado favorável para sua comercialização; entretanto, tais condições dificilmente estão todas presentes em determinado sistema de produção. Torna-se imprescindível a busca de novas alternativas de cultivo e tecnologia para aumento da produtividade e maior estabilidade de produção, tornando o setor agrícola competitivo e auto-sustentável.

Uma das técnicas da agricultura moderna muito utilizada para viabilizar o cultivo fora de época, diminuir custos e aumentar a produtividade, é o cultivo protegido que, juntamente com as novas tecnologias aplicadas à área de irrigação, como a fertirrigação, tem propiciado bons resultados (Factor et al., 2008). A irrigação e a adubação são considerados importantes fatores a influenciar a produtividade e, quando aplicadas juntas, influenciam o desenvolvimento das plantas, a produção e a qualidade dos frutos (Bar-Yosef, 1999). Uma das vantagens mais significativas da fertirrigação está relacionada à eficiência de absorção de nutrientes pela planta, por torná-los prontamente disponíveis na solução do solo, fáceis de serem absorvidos (Marcussi, 2005).

Entre as culturas com relevância econômica cultivada em ambiente protegido, se destaca o pimentão. Segundo Melo (1997), é a cultura que melhor tem se adaptado a esta condição de cultivo. Lorentz et al. (2002) mencionam que das hortaliças cultivadas em ambiente protegido o pimentão se situa entre as cinco culturas com maior área cultivada, não só no Brasil mas também em diversos países devido à grande produtividade e qualidade dos frutos que podem ser alcançadas nessas condições.

O nitrogênio se sobressai entre os principais nutrientes ligados ao aumento de produtividade do pimentão, em razão de desempenhar papel fundamental no crescimento e no rendimento da cultura; no entanto, o manejo adequado da adubação nitrogenada em ambiente protegido assume papel proeminente e diverso daquele verificado a campo, exigindo manejo diferenciado pois, em ambiente protegido, o ciclo da cultura é mais longo e, com a utilização de fertirrigação, pode haver acúmulo de sais no perfil do solo, influenciando a absorção de N pelo pimentão (Moreno et al., 1996).

Sobretudo em relação ao nitrogênio, é necessário definir quanto aplicar em cada fertirrigação para atender à demanda da planta. A redução na produção de pimentão em resposta ao aumento da dose de sais nitrogenados tem sido associada ao aumento do potencial osmótico da solução do solo (Fontes et al., 2005): já a deficiência desse nutriente, segundo Marcussi et al. (2004), provoca redução na produtividade e qualidade dos frutos. Estes autores verificaram que, quando a cultura de pimentão foi fertirrigada com nitrogênio, as plantas que receberam a maior dose (15,2 g $\mathrm{N}$ por planta) atingiram maior produtividade e maior peso médio de frutos. Villas Bôas (2001) obteve maior produção de frutos comerciais de pimentão com a aplicação de 16,5 g N por planta.

Embora sejam poucos os trabalhos de pesquisa publica- dos no Brasil sobre fertirrigação de hortaliças, esta prática é bastante difundida principalmente entre os horticultores que utilizam a irrigação por gotejamento; portanto, é notória a necessidade de mais informações quanto às quantidades de nitrogênio a serem aplicadas em ambiente protegido, para que se possa obter rendimento satisfatório da variedade utilizada, evitando-se aplicações elevadas quando desnecessárias ou que possam ter efeito depressivo.

Nesse sentido objetivou-se, com este trabalho, estudar os aspectos relacionados às características de frutos de pimentão cultivado sob diferentes doses de nitrogênio aplicadas via fertirrigação, em ambiente protegido.

\section{MATERIAL E MÉTODOS}

O trabalho foi conduzido nos meses de fevereiro a junho de 2004, no Centro de Ciências Agrárias, UFPB, município de Areia, PB, na Microrregião do Brejo Paraibano, a $60^{\circ} 58^{\prime} 12$ ' de Latitude Sul e $350^{\circ} 42^{\prime} 15^{\prime}$ ' de Longitude Oeste de Greenwinch, com altitude de aproximadamente 575 m (Gondim \& Fernandez, 1980).

O delineamento experimental foi em blocos casualizados, com cinco tratamentos $\left(0,100,200,300\right.$ e $400 \mathrm{~kg} \mathrm{ha}^{-1}$ de N) e quatro repetições. Em cada parcela experimental com dimensões de 2,40 x 2,10 m (5,04 $\left.\mathrm{m}^{2}\right)$ foram transplantadas mudas de pimentão, em duas filas duplas, seguindo-se um modelo triangular, perfazendo o total de 18 plantas por parcela, distanciadas $50 \mathrm{~cm}$, sendo o espaço entre cada fila dupla de $80 \mathrm{~cm}$. O transplantio foi feito em fevereiro de 2004.

Utilizou-se uma estufa plástica, em arco, com $189 \mathrm{~m}^{2}$, altura na lateral livre 3,00 m, coberta com filme plástico de $120 \mathrm{~mm}$ e fechamento lateral com tela para sombreamento de 30\%, plástico para "saia” com $40 \mathrm{~cm}$ de altura e cortinas móveis manuais para laterais e fundo.

O solo da área experimental foi classificado como Neossolo Regolítico Psamitico Típico, textura areia franca, com as seguintes características químicas: $\mathrm{pH}\left(\mathrm{H}_{2} \mathrm{O}\right)$ : 6,2; $\mathrm{P}$ (mg dm$\left.{ }^{-3}\right): 184,43 ; \mathrm{K}^{+}\left(\mathrm{mg} \mathrm{dm}^{-3}\right): 26,10 ; \mathrm{Na}^{+}\left(\mathrm{cmol} \mathrm{dm}^{-3}\right)$ : 0,07; $\mathrm{H}^{+}+\mathrm{Al}^{+3}\left(\mathrm{cmol} \mathrm{dm}^{-3}\right): 0,25 ; \mathrm{Al}^{+3}\left(\mathrm{cmol} \mathrm{dm}^{-3}\right): 0,00 ; \mathrm{Ca}^{+2}$ $\left(\mathrm{cmol} \mathrm{dm}{ }^{-3}\right): 2,65 ; \mathrm{Mg}^{+2}\left(\mathrm{cmol} \mathrm{dm}^{-3}\right): 1,50 ; \mathrm{SB}\left(\mathrm{cmol} \mathrm{dm}^{-3}\right)$ : 4,29; CTC (cmol dm$\left.{ }^{-3}\right)$ : 4,54; M.O (g dm$\left.{ }^{-3}\right)$ : 5,28.

Fez-se, durante o preparo das parcelas, uma adubação em fundação com esterco bovino, na quantidade de $20 \mathrm{t} \mathrm{ha}^{-1}$, incorporado ao solo 10 dias antes do transplantio, de acordo com a recomendação laboratorial. Os resultados da análise química do esterco, foram: $\mathrm{N}\left(\mathrm{g} \mathrm{kg}^{-1}\right)$ : 14,53; $\mathrm{P}\left(\mathrm{g} \mathrm{kg}^{-1}\right)$ : 4,52; e $\mathrm{K}\left(\mathrm{g} \mathrm{kg}^{-1}\right)$ : 15,86; as doses de nitrogênio, foram aplicadas via fertirrigação e parceladas em sete aplicações, a cada 10 dias. Aplicou-se o potássio recomendado (120 kg ha-1) simultaneamente com o nitrogênio, também via fertirrigação e em todos os tratamentos, inclusive na testemunha. Não se utilizou fósforo em virtude da alta quantidade presente no solo, visto que a área vinha sendo cultivada com outras hortaliças.

A variedade utilizada foi a All Big, pertencente ao grupo conhecido como Cascadura, muito cultivada na região do Brejo Paraibano; o sistema de irrigação foi por gotejamento, 
sendo cada fileira de planta irrigada com uma tubulação de distribuição contendo um gotejador por planta com vazão média de 4,0 L h${ }^{-1}$; realizou-se o cálculo da lâmina de irrigação com base na disponibilidade de água do solo. O turno de rega foi de um dia, com tempo de irrigação de $25 \mathrm{~min}$. A dosagem de fertilizante foi 64, 128, 192 e 256 g de uréia por aplicação, para as doses de 100, 200, 300 e $400 \mathrm{~kg} \mathrm{ha}^{-1}$ de $\mathrm{N}$, respectivamente. A água utilizada provinha de um poço Amazonas e classificada como $\mathrm{C}_{1} \mathrm{~S}_{1}$ conforme United States Salinity Laboratory (USSL), (Ayers \& Westcot, 1991). Efetuaram-se, durante o ciclo da cultura, quatro capinas manuais para eliminar ervas daninhas e tutoramento para sustentação das plantas.

Os frutos foram classificados pelo tamanho, de acordo com as normas vigentes no Ministério da Agricultura (Brasil, 2001), da seguinte forma: comerciais (comprimento $\geq 60 \mathrm{~mm}$ e diâmetro $\geq 40 \mathrm{~mm}$ ) e não comerciais (comprimento $<60$ mm e diâmetro < 40 mm ou algum defeito grave, tais como: frutos murchos, deteriorados, malformados, com danos mecânicos, por doenças ou pragas). Avaliaram-se: comprimento e diâmetro dos frutos, em que o comprimento foi obtido medindo-se o fruto do ápice à base e o diâmetro medido na região central, com uso de um paquímetro digital; o peso médio dos frutos foi obtido com a pesagem dos frutos colhidos em cada tratamento, dividido pelo número total de frutos do tratamento; o número de frutos por planta (total, comercial e não-comercial) correspondeu ao número total de frutos dividido pelo número de plantas de cada tratamento.

Os resultados foram submetidos a análise de variância (pelo teste $\mathrm{F}$ a 5\% de probabilidade) e de regressão, através do Software SISVAR (Sistema para Análises Estatísticas) desenvolvido pela Universidade Federal de Lavras.

\section{RESULTADOS E DISCUSSÃO}

As variações de temperatura e umidade relativa do ar, observadas dentro da estufa durante a condução do experimento, são apresentadas na Figura 1.

Verificou-se tendência de diminuição da temperatura na medida em que se aproximava o período chuvoso. As temperaturas máximas, acima de $30^{\circ} \mathrm{C}$ ocorreram com freqüência até aproximadamente os 90 dias Juliano; posteriormente, até o final da colheita aos 154 dias Juliano, essas temperaturas se situaram próximas ou inferiores a $30^{\circ} \mathrm{C}$ e as mínimas não inferiores a $20^{\circ} \mathrm{C}$ (Figura 1A); a temperatura média oscilou entre 25 a $30^{\circ} \mathrm{C}$. Segundo Sganzerla (1997) a temperatura ideal para o pimentão deve estar entre 20 e $25^{\circ} \mathrm{C}$. Outros autores, como Pádua et al. (1984) e Pereira (1990) concordam que a temperatura do ar deva variar entre 16 e $30^{\circ} \mathrm{C}$.

A amplitude térmica entre o dia e a noite ao longo do período avaliado oscilou entre 7 a $10^{\circ} \mathrm{C}$, faixa considerada ideal para a cultura, segundo Goto \& Tivelli (1998). Verificou-se que, à noite, a temperatura permaneceu mais elevada, em média $1,14^{\circ} \mathrm{C}$, no ambiente protegido em relação ao ambiente externo, sendo este fator positivo para o crescimento e desenvolvimento das plantas e, como conseqüência, para a produção.
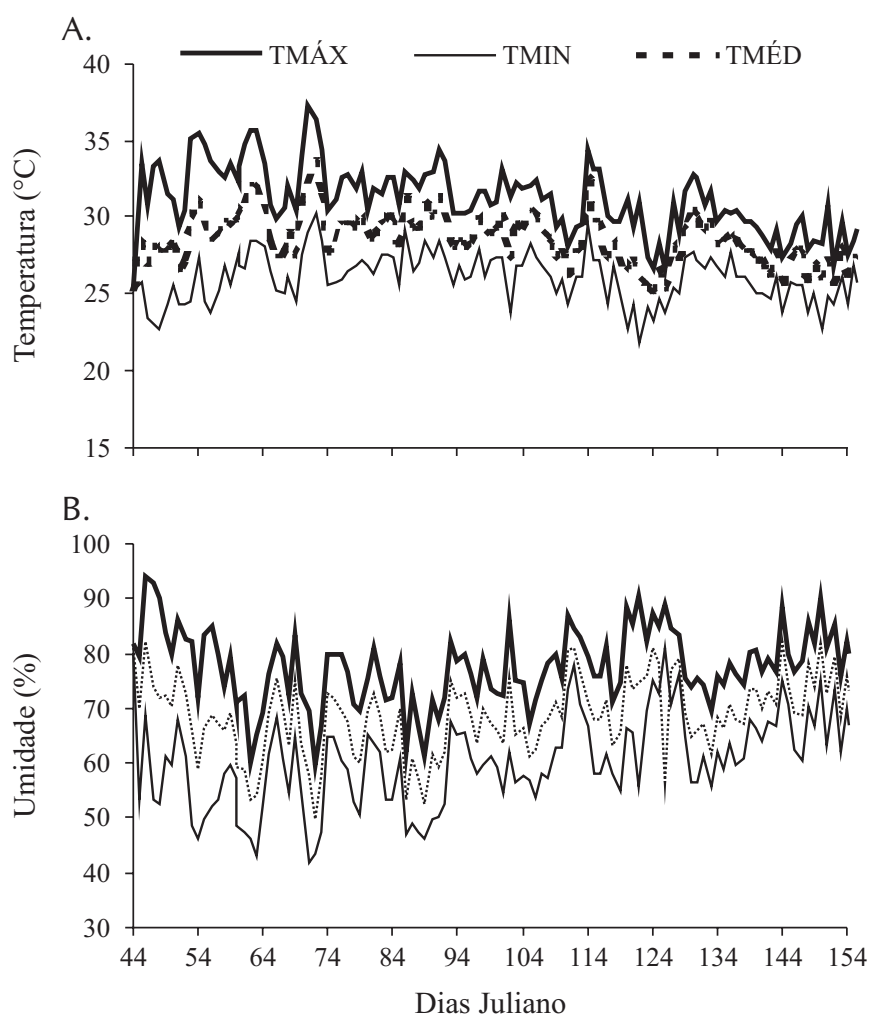

Figura 1. Evolução da temperatura (A) e da umidade relativa do ar (UR) (B), dentro da estufa, expressa em valores máximos, mínimos e médios, medidas no período de 13/02/2004 a 07/06/2004

Houve elevação gradativa da umidade no interior da estufa (Figura 1B) sempre que a temperatura diminuía. Os valores médios de umidade observados no interior da estufa encontravam-se ligeiramente acima da faixa considerada ideal para a cultura, que segundo Goto \& Tivelli (1998) é de 50 a $70 \%$.

Na Tabela 1 se encontram os resultados da análise de variância e de regressão para comprimento (COMP), diâmetro (DAM) e peso médio (PM) de frutos de pimentão cultivar All Big, em função das doses de nitrogênio aplicadas via fertirrigação.

Tabela 1. Resumo da análise de variância e de regressão para comprimento (COMP), diâmetro (DAM) e peso médio (PM) de frutos de pimentão cultivar All Big, em função das doses de nitrogênio, aplicadas via fertirrigação

\begin{tabular}{lcccc}
\hline \multirow{2}{*}{ Fontes de variação } & G.L. & \multicolumn{3}{c}{ Quadrado Médio } \\
\cline { 3 - 5 } & & COMP. & DAM & PM \\
Blocos & 3 & $4,269^{\text {NS }}$ & $1,558^{\text {NS }}$ & $2,670^{\text {NS }}$ \\
Níveis de N & 4 & $7,426^{\text {NS }}$ & $12,667^{* *}$ & $126,471^{\text {** }}$ \\
Linear & 1 & - & $33,800^{* *}$ & $377,610^{* *}$ \\
Quadrática & 1 & - & $5,856^{\text {NS }}$ & $76,191^{\text {NS }}$ \\
Resíduo & 12 & 5,189 & 1,494 & 16,975 \\
\hline CV (\%) & & 2,77 & 2,09 & 5,00 \\
\hline
\end{tabular}

O comprimento médio de frutos foi de $82 \mathrm{~mm}$ e não foi influenciado pelas doses de nitrogênio; já o diâmetro (Figura 2A) e o peso médio de frutos (Figura 2B) aumentaram $(\mathrm{P}<0,01)$ toda vez em que essas doses foram maiores. 
A.

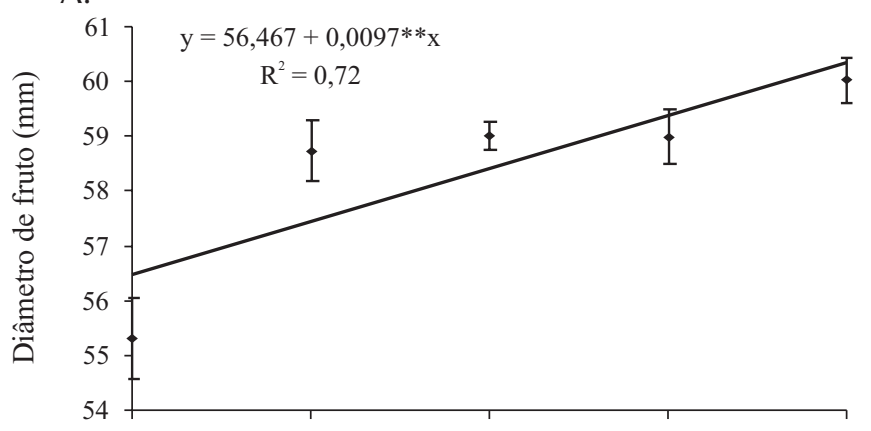

B.

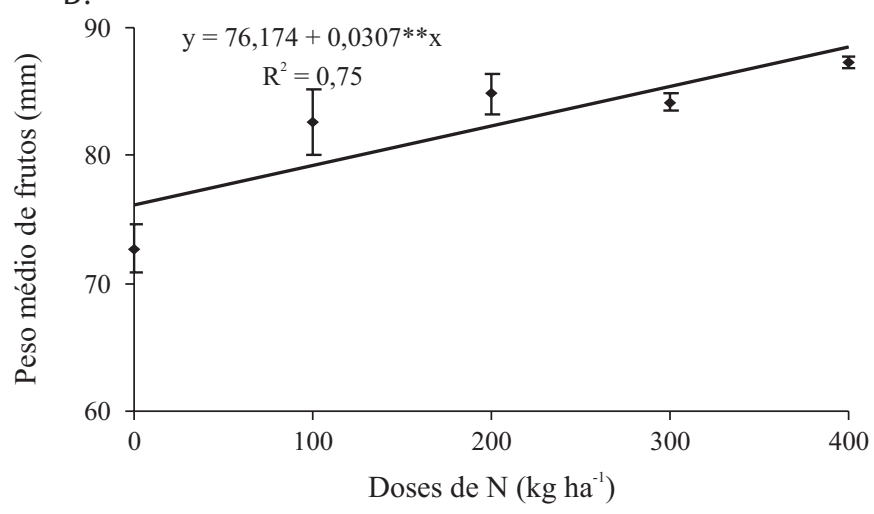

Figura 2. Diâmetro (A) e peso médio de frutos (B) de pimentão, variedade All Big, em função das doses de nitrogênio aplicadas via fertirrigação; cada ponto representa a média de quatro repetições; as barras indicam o desvio padrão da média

Resultados semelhantes foram encontrados por Paes (2003), cuja aplicação de NPK influenciou o diâmetro e o peso médio de frutos, mas não o comprimento, enquanto Goyal et al. (1989) relatam o efeito positivo do N para todas essas características. Os valores obtidos para o comprimento de frutos estão dentro da média padrão $(8-13 \mathrm{~cm})$ para essa variedade (Isla, 2004). Com a dose máxima de nitrogênio (400 kg ha-1), o diâmetro de frutos obtido foi de $60 \mathrm{~mm}$ enquanto na testemunha o valor foi de $56,5 \mathrm{~mm}$. O maior peso médio de frutos, de aproximadamente $90 \mathrm{~g}$, foi constatado na dose máxima de nitrogênio e o menor, de 77 g, na testemunha. Marcussi \& Villas Boas (2003) também verificaram efeito benéfico do nitrogênio sobre esta característica. Esses resultados podem estar relacionados com a forma de aplicação do $\mathrm{N}$, ou seja, com o uso da fertirrigação, em que os nutrientes são aplicados em doses e tempo apropriados para o específico estágio de desenvolvimento das plantas, o que pode ser confirmado na literatura, na qual há trabalhos que demonstram melhores resultados da fertirrigação em relação à adubação convencional para essas características (Villas Boas et al., 2000; Leite Júnior, 2001).

Já Blanco \& Folegatti (2008) avaliando a produção e a qualidade de frutos de tomate, sob diferentes doses de $\mathrm{N}$ e K aplicados via fertirrigação por gotejamento, não verificaram efeitos dos tratamentos sobre os componentes de produção (produtividade, tamanho e peso médio dos frutos).

Na Tabela 2 estão os resultados da análise de variância e de regressão para número total (NFT), comercial (NFC) e nãocomercial (NFNC) de frutos de pimentão cultivar All Big, em
Tabela 2. Resumo da análise de variância e de regressão para número de frutos por planta, total (NFT), comercial (NFC) e não-comercial (NFNC) de pimentão cultivar All Big, em função das doses de nitrogênio, aplicadas via fertirrigação

\begin{tabular}{lcccc}
\hline \multirow{2}{*}{ Fontes de variação } & \multirow{2}{*}{ G.L. } & \multicolumn{3}{c}{ Quadrado Médio } \\
\cline { 3 - 5 } Blocos & 3 & NFT & NFC & NFNC \\
Níveis de N & 4 & $45,060^{\text {NS }}$ & $7,976^{\text {NS }}$ & $0,848^{\text {NS }}$ \\
Linear & 1 & $163,499^{* *}$ & $125,331^{* *}$ & $1,267^{* *}$ \\
Quadrática & 1 & $7,927^{\text {NS }}$ & $4,066^{\text {NS }}$ & $0,102^{\text {NS }}$ \\
Resíduo & 12 & 4,802 & $3,875^{* *}$ \\
\hline CV (\%) & & 18,95 & 20,33 & 0,687 \\
\hline
\end{tabular}

função das doses de nitrogênio, aplicadas via fertirrigação.

O número total (Figura 3A) e comercial (Figura 3B) de frutos aumentou em função das doses de nitrogênio $(P<0,01)$ enquanto o número de frutos não-comercial diminuiu com o

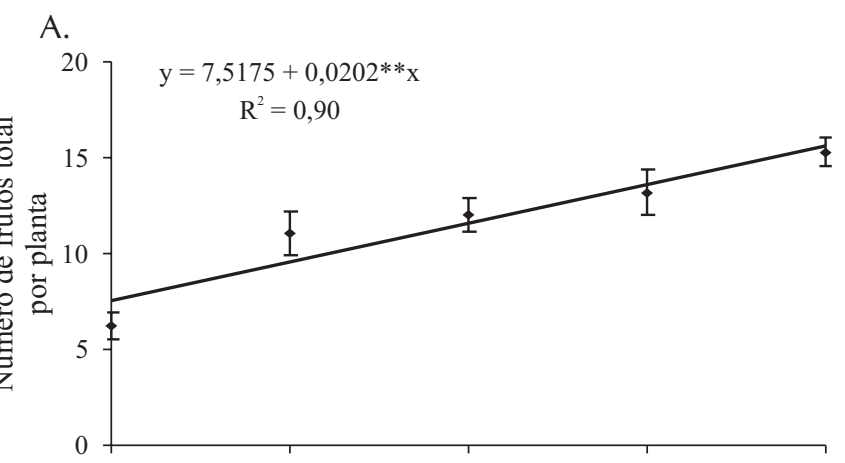

B.

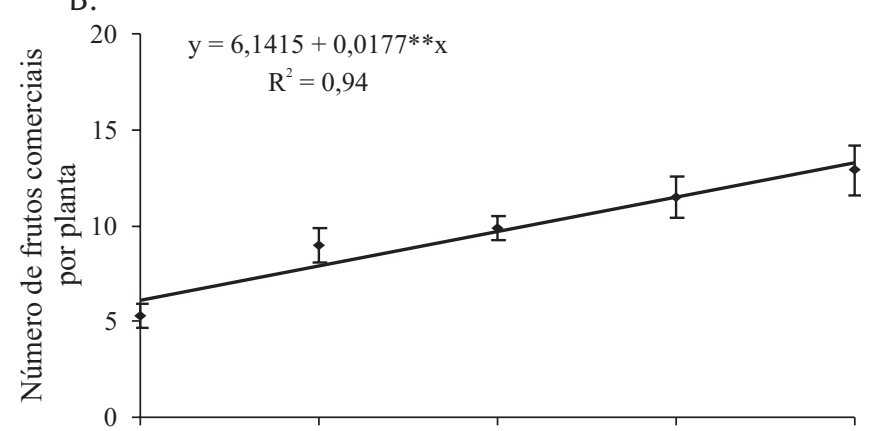

C.

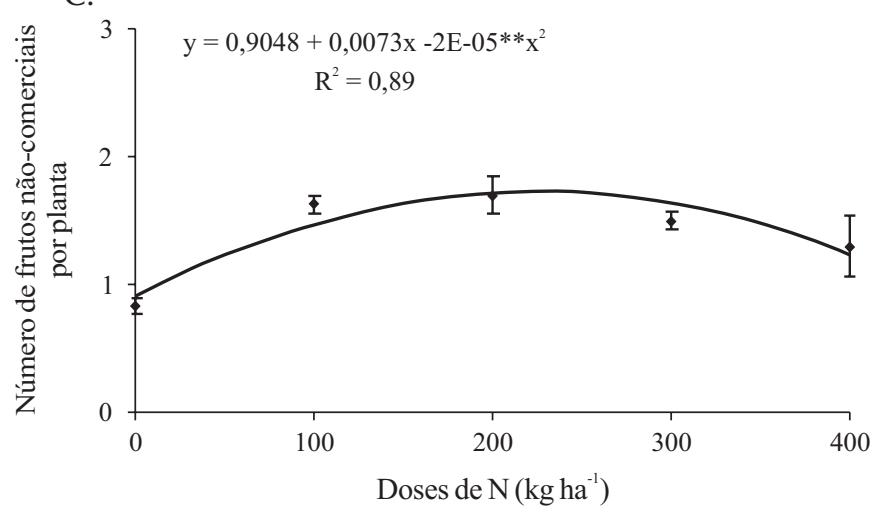

Figura 3. Número de frutos total (A), comercial (B) e não comercial (C) de pimentão, variedade All Big, em função das doses de nitrogênio aplicadas via fertirrigação; cada ponto representa a média de quatro repetições; as barras indicam o desvio padrão da média 
aumento dessas doses $(\mathrm{P}<0,01)$, a partir de $200 \mathrm{~kg} \mathrm{ha}^{-1}$ de nitrogênio (Figura $3 \mathrm{C}$ ).

O número máximo de frutos por planta foi de 15,27 , para o total e 12,80 para o comercial, observado na dose máxima de nitrogênio. Na testemunha esses valores foram de 6,2 (total) e 5,2 (comercial). Marcussi \& Villas Boas (2003), avaliando a eficiência de aproveitamento de $\mathrm{N}$ e K pelo pimentão sob fertirrigação, obtiveram média de 13 frutos por planta em cultivo protegido. Em condições de cultivo em campo aberto, no Brejo Paraibano, Leite Júnior (2001) obteve média de 7,5 frutos por planta e Paes (2003) de 9,0 frutos por planta.

O número de frutos não-comercial ajustou-se a um modelo de regressão quadrático (Figura $3 \mathrm{C}$ ), cujo número máximo de frutos (1,57 frutos) foi obtido na dose de $182,5 \mathrm{~kg} \mathrm{ha}^{-1}$ de nitrogênio, sendo o valor de $\mathrm{R}^{2}=0,89$; no tratamento com a maior dose de $\mathrm{N}$ o valor encontrado de 9,6\% de frutos nãocomerciais se acha próximo ao verificado por Leonardo (2003).

Os frutos considerados não-comerciais se apresentavam com excelente aspecto, em termos de aparência e qualidade sanitária, tendo apenas comprimento e diâmetro fora dos padrões preestabelecidos.

\section{CONCLUSÕES}

1. O comprimento de frutos e o número de frutos nãocomercial do pimentão não foram influenciados pelas doses de nitrogênio.

2. Diâmetro, peso médio e número de frutos total e comercial do pimentão, foram influenciados de forma linear e positiva pelas doses de nitrogênio.

3. O número de frutos por planta não-comercial foi influenciado pelas doses de nitrogênio, de forma quadrática, tendo o número máximo de frutos $(1,57)$ ocorrido para a dose de $182,5 \mathrm{~kg} \mathrm{ha}^{-1}$.

4. A dose máxima de nitrogênio (400 $\left.\mathrm{kg} \mathrm{ha}^{-1}\right)$ promoveu maior número de frutos comerciais (12,8 frutos por planta), em relação à testemunha $\left(0 \mathrm{~kg} \mathrm{ha}^{-1} \mathrm{de} \mathrm{N}, 5,5\right.$ frutos por planta) representando aumento de $133 \%$.

\section{LITERATURA CITADA}

Ayers, R. S.; Westcot, D. W. A qualidade da água na agricultura. Campina Grande: UFPB, 1991. 218p. Estudos da FAO, Irrigação e Drenagem, 29.

Bar-Yosef, B. Advances in fertigation. In: Sparks, D. L. (ed.) Advances in agronomy. New York: Academic Press, 1999. p.1-77.

Blanco, F. F; Folegatti, M. V. Doses de N e K no tomateiro sob estresse salino: III. Produção e qualidade de frutos. Revista Brasileira de Engenharia Agrícola e Ambiental, v.12, n.2, p.122-127, 2008.
Brasil. Ministério da Agricultura. Legislação aplicada à agricultura: Classificação de produtos vegetais. Artigo $3^{\circ}$. Classificação do pimentão. Disponível em: http://agridata.mg.gov.br/legislacao/classificacao_cer.../laclassprodvegetalpimentao.nt>. 27 Set. 2001.

Factor, T. L.; Araújo, J. A. C. de; Vilella Júnior, V. E. Produção de pimentão em substratos e fertirrigação com efluente de biodigestor. Revista Brasileira de Engenharia Agrícola e Ambiental, v.12 n.2, p.143-149, 2008.

Fontes, P. C. R; Dias, E. N.; Graça, R. N. Acúmulo de nutrientes e método para estimar doses de nitrogênio e de potássio na fertirrigação do pimentão. Horticultura Brasileira, v.23, n.2, p.275-280, 2005.

Gondim, A. W. A.; Fernandez, B. Probabilidade de chuvas para 0 município de Areia-PB. Agropecuária Técnica, v.1, n.1, p.5563, 1980.

Goto, R.; Tivelli, S. W. (Org.). Produção de hortaliças em ambiente protegido: condições subtropicais. São Paulo: UNESP, 1998. 319p.

Goyal, M. R.; Luna, R. G.; Hernández, E. R. de; Baéz, C. C. de. Pos-harvest evaluation of nitrogen fertigated sweet peppers under drip irrigation and plastic mulch. Journal of Agriculture of University of Puerto Rico, v.73, n.2, p.109-115, 1989.

Isla Sementes Ltda. Pimentão All Big. http://www.isla.com.br/cgibin/detalhe.cgi?id=206. 9 Out. 2004.

Leite Júnior, G. P. Redução ou aumento das doses de nitrogênio e potássio aplicadas ao pimentão via fertirrigação à adubação convencional. Areia: UFPB, 2001. 65p. Dissertação Mestrado.

Leonardo, M. Estresse salino induzido em plantas de pimentão (Capsicum annuum L.) fertirrigadas e seus efeitos sobre a produtividade e parâmetros bioquímicos. Botucatu: UNESP, 2003. 100p. Dissertação Mestrado

Lorentz, L. H.; Lúcio, A. D.; Heldwein, A. B.; Souza, M. F.; Mello, R. M. Estimativa da amostragem para pimentão em estufa plástica. Horticultura Brasileira, v.20, n.2, 2002. Suplemento 2. CD Rom.

Marcussi, F. F. N.; Villas Bôas, R. L. Uso da fertirrigação na eficiência de aproveitamento de $\mathrm{N}$ e K pelo pimentão sob condições de cultivo protegido. In: Congresso Brasileiro de Fertirrigação, 1, 2003, João Pessoa. Anais...João Pessoa: UFPB, [s.n.], 2003. CD Rom.

Marcussi, F. F. N.; Godoy, L. J. G. de; Villas Boas, R. L. Fertirrigação nitrogenada e potássica na cultura do pimentão baseada no acúmulo de n e k pela planta. Irriga, v.9, n.1, p 41-51, 2004.

Marcussi, F. F. N. Uso da fertirrigação e teores de macronutrientes em planta de pimentão. Engenharia Agrícola, v.25, n.3, p.642-650, 2005.

Melo, A. M. T. Análise genética de caracteres de frutos em híbridos de pimentão. Piracicaba: ESALQ/USP, 1997. 112p. Tese Doutorado

Moreno, D. A.; Pulgar, G.; Villora, G.; Romero, L. Effect of N and $\mathrm{K}$ on fruit production and leaf levels of $\mathrm{Fe}, \mathrm{Zn}, \mathrm{Cu}$ and $\mathrm{B}$ and their biochemical indicator in capsicum plants. Phyton, v.59, n.1-2, p.1-12, 1996. 
Pádua, J. G; Casali, V. W. D.; Pinto, C. M. F. Efeitos climáticos sobre pimentão e pimenta. Informe Agropecuário, v.10, n.113, p.11-13, 1984.

Paes, R. de A. Rendimento do pimentão (Capsicum annuum L.) cultivado com urina de vaca e adubação mineral. Areia: UFPB, 2003. 65p. Dissertação Mestrado

Pereira. A. L. Cultura do pimentão. Fortaleza: DNOCS, 1990. 49p. Sganzerla, E. Nova agricultura: A fascinante arte de cultivar com os plásticos. 6.ed. Guaíba: Agricultura, 1997. 342p.
Villas Boas, R. L. Doses de nitrogênio para o pimentão aplicadas de forma convencional e através da fertirrigação. Botucatu: UNESP, 2001. 123p. Tese Doutorado

Villas Boas, R. L.; Kano, C.; Lima, C. P.; Nanetti, F. A.; Fernandes. D. M. Efeito de doses de nitrogênio aplicado de forma convencional e através da fertirrigação na cultura do pimentão. Horticultura Brasileira, v.18, p.801-802, 2000. Suplemento. 